\title{
An Efficient Pattern Search Method
}

\author{
Xiaoli Zhang, Qinghua Zhou, Yue Wang \\ College of Mathematics and Computer Science, Hebei University, Baoding, 071002, Hebei, China \\ Email: zxl.hbu@gmail.com, qinghua.zhou@gmaill.com
}

Received June 2013

\begin{abstract}
Pattern search algorithms is one of most frequently used methods which were designed to solve the derivative-free optimization problems. Such methods get growing need with the development of science, engineering, economy and so on. Inspired by the idea of Hooke and Jeeves, we introduced an integer $m$ in the algorithm which controls the number of steps of iteration update. We mean along the descent direction, we allow the algorithm to go ahead $\mathrm{m}$ steps at most to explore whether we can get better solution further. The experiment proved the strategy's efficiency.
\end{abstract}

Keywords: Unconstrained Optimization; Derivative-Free Optimization; Pattern Search Methods; Positive Bases

\section{Introduction}

In this paper, we consider the unconstrained minimization problem

$$
\min _{x \in R^{n}} f(x)
$$

where $f: R^{n} \rightarrow R$, is continuously differentiable, but the information about the gradient of $f$ is either unavailable or unreliable. There are lots of problems where derivatives are unavailable but we also want to do some optimizations. The diversity of applications comes from different complicated backgrounds with economics, engineering, mathematics, finance, and so on (see [1-3] for instance).

In such cases, derivative-free optimization methods (also named direct search methods) which neither compute nor approximate derivatives play an important role. The reader is referred to see [4-6]. In [5], the author introduced an ingenious idea for a generalized pattern search method and gave convergence analysis. It includes several known algorithms as its special cases. Familiar with the analysis of the property of the generalized method, the author developed two new classes of pattern search methods [6].

Inspired by the idea of Hooke and Jeeves [7], we improved the method of [6] by introducing an integer $m$.

We mean, if a step is successful (the value of $f$ decrease), then the same direction maybe also be proved successfully at the current point. So, we allow the algorithm to explore the same direction further. On the other hand, if it always goes ahead along one direction until it can not improve the value of $f$ any more, it likely neglects additional information which other directions can offer. To balance these two aspects, we introduce an integer $m$ be used to control iteration steps which we mean that we allow the algorithm to iterate at most $m$ steps along the same direction.

Next, we would like to present some basic concepts we need.

\section{Pattern Search Methods and Positive Bases}

We use $\|\cdot\|$ and $\langle\cdot, \cdot\rangle$ to represent the Euclidean norm and inner product, respectively. By abuse of notation, if $A$ is a matrix, $a \in A$ means that the vector $a$ is a column of $A$. It will also be convenient to assume that $\left[a_{1}, a_{2}, \cdots, a_{r}\right]$ represents, not only the matrix with $r$ columns, but also, depending on the context, the set of $r$ vectors $\left[a_{1}, a_{2}, \cdots, a_{r}\right]$. The identity matrix is denoted by $I$ and its $i$-th column by $e_{i}$. Finally, we write $e$ to represent a vector of ones with appropriate size.

\subsection{Positive Bases}

We present a few basic properties of positive bases beginning from the theory of positive linear dependence developed by Davis [8]. The positive span of a set of vectors $\left[v_{1}, v_{2}, \cdots, v_{r}\right]$ is the convex cone

$$
\left\{v \in R_{n}: v=\sum_{i=1}^{r} a_{i} v_{i}, \alpha_{i} \geq 0, i=1,2, \cdots, r\right\}
$$

The set $\left[v_{1}, v_{2}, \cdots, v_{r}\right]$ is said to be positively dependent if one of the vectors is in the convex cone positively spanned by the remaining vectors, i.e., if one of the vectors is a positively combination of the others; otherwise the set is positively independent. A positive basis is 
a positively independent set whose positive span is $R^{n}$. Alternatively, a positive basis for $R^{n}$ can be defined as a set of nonzero vectors of $R^{n}$ whose positive combinations span is $R^{n}$, but no proper set does. The following theorem in [8] indicates that a positive spanning set contains at least $n+1$ vectors in $R^{n}$.

Theorem 1 If $\left[v_{1}, v_{2}, \cdots, v_{r}\right]$ positively spans $R^{n}$, then it contains a subset with $r-1$ elements that spans $R^{n}$.

Furthermore, a positive basis can not contain more than $2 n$ elements ([8]). Positive basis with $n+1$ and $2 n$ elements are referred to as minimal and maximal positive basis respectively.

We present now three necessary and sufficient characterizations for a set of vectors that spans $R^{n}$ or spans $R^{n}$ positively ([8]).

Theorem 2 Let $\left[v_{1}, v_{2}, \cdots, v_{r}\right]$, with $v_{i} \neq 0$ for all $i=1,2, \cdots, r$, span $R^{n}$. Then the following are equivalent: i) $\left[v_{1}, v_{2}, \cdots, v_{r}\right]$ positively spans for $R^{n}$. ii) For every $i=1,2, \cdots, r,-v_{i}$ is in the convex cone positively spanned by the remaining $r-1$ vectors. iii) There exist real scalars $\alpha_{1}, \alpha_{2}, \cdots, \alpha_{r}$ with $\alpha_{i}>0$, $i \in\{1, \cdots, r\}$, such that $\sum_{i=1} \alpha_{i} v_{i}=0$. iv) For every nonzero vector $b \in R^{n}$, there exists an index $i$ in $i \in\{1, \cdots, r\}$ for with $b^{T} v_{i}>0$.

The following result provides a simple mechanism for generating different positive bases. The proof can be found in [6].

Theorem 3 Suppose $\left[v_{1}, v_{2}, \cdots, v_{r}\right]$ is a positive basis for $R^{n}$ and $B \in R^{n \times n}$ is a nonsingular matrix, then $\left[B v_{1}, B v_{2}, \cdots, B v_{r}\right]$ is also a positive basis for $R^{n}$.

From above theorems, we can easily deduce the following corollary.

Corollary 1 Let $B=\left[b_{1}, b_{2}, \cdots, b_{n}\right] \in R^{n \times n}$ be a nonsingular matrix, then $\left[B,-\sum_{i=1}^{n} b_{i}\right]$ is a positive basis for $R^{n}$.

A trivial consequence of this corollary is that $[I, e]$ is a positive basis.

\subsection{Pattern Search Methods}

Pattern search methods are characterized by the nature of the generating matrices and the exploratory moves algorithms. These features are discussed more fully in [5] and [9].

To define a pattern, we need two components, a basis matrix and a generating matrix.

The basis matrix can be any nonsingular matrix $B \in R^{n \times n}$. The generating matrix is a matrix

$C_{k} \in Z^{n \times P_{k}}$, where $P_{k}>n+1$. We partition the generating matrix into components $C_{k}=\left[\Gamma_{k}, L_{k}, 0\right]$

We require that $\Gamma_{k} \in M$, where $M$ is a finite set of integral matrices with full row rank. We will see that $\Gamma_{k}$ must have at least $n+1$ columns. The 0 in the last column of $C_{k}$ is a single column of zeros.

A pattern $P_{k}$ is then defined by the columns of the matrix $P_{k}=B C_{k}$. For convenience, we use the partition of the generating matrix $C_{k}$ to partition $P_{k}$ as follows:

$$
P_{k}=B C_{k}=\left[B \Gamma_{k}, B L_{k}, 0\right]
$$

Given $\Delta_{k} \in R, \Delta_{k}>0$, we define a trial step $s_{k}^{i}$ to be any vector of the form $s_{k}^{i}=\Delta_{k} B C_{k}^{i}$, where $C_{k}^{k}$ is a to be any vector of the form $s_{k}^{i}=\Delta_{k} B C_{k}^{i}$, column of $C_{k}$. Note that $B C_{k}^{i}$ determines the direction of the step, while $\Delta_{k}$ serves as a step length parameter.

At the $k$-th iteration process, we define a trial point as any point of the form $x_{k}^{i}=x_{k}+s_{k}^{i}$, where $x_{k}$ is the current iteration point.

The following algorithms state the pattern search method for unconstrained minimization.

\subsection{Algorithm 1 Pattern Search Method}

Let $x_{0} \in R_{n}$ and $\Delta_{0}>0$ be given.

For $k=0,1,2, \cdots$

Compute $f\left(x_{k}\right)$.

Determine a step $s_{k}$ using an unconstrained exploratory moves algorithm.

If $f\left(x_{k}+s_{k}\right)<f\left(x_{k}\right)$, then $x_{k+1}=x_{k}+s_{k}$, otherwise $x_{k+1}=x_{k}$.

Update $C_{k}$ and $\Delta_{k}$.

\subsection{Algorithm 2 Updating $\Delta_{k}$}

$$
\begin{aligned}
& \text { Let, and } \begin{array}{r}
\left\{w_{0}, w_{1}, \cdots, w_{l}\right\} \subset Z, \\
w_{0}<0 \quad, w_{i} \geq 0, i=1,2, \cdots, l
\end{array} \\
& \text { Let } \theta=\tau^{w_{0}}, \quad \lambda_{k} \in\left\{\tau^{i}, i=1,2, \cdots, l\right\} . \\
& \text { If } f\left(x_{k}+s_{k}\right) \geq f\left(x_{k}\right), \text { then } \Delta_{k+1}=\theta \Delta_{k} . \\
& \text { If } f\left(x_{k}+s_{k}\right)<f\left(x_{k}\right) \text {, then } \Delta_{k+1}=\lambda_{k} \Delta_{k} .
\end{aligned}
$$

\section{Our Algorithm and Numerical Results}

In [5], the generating matrix has the form $C_{k}=\left[M_{k},-M_{k}, L_{k}, 0\right]$ for some $n \times n$ nonsingular matrix $M_{k}$. In light of the above discussion, the nature of $\Gamma_{k}=\left[M_{k},-M_{k}\right]$ as a maximal positive basis is now revealed.

In [6], the author reduced the number of objective evaluations in the worst case from $2 n$ to as few as $n+1$. The choice is to make $\Gamma_{k}$ include $n+1$ columns which are just the minimal positive bases.

In this paper, we simply select the relative parameters as follows: $B=I, \Gamma_{k}=[I,-e]$ with $e=(1,1, \cdots, 1)^{T}$,

$$
\theta=1 / 2, \lambda_{k}=1
$$

Then, we have all we need to state our algorithm now. 


\section{Algorithm 3 Modified Pattern Search Method}

(1) Start with $x_{0}, \Delta_{0}, f_{0}, k=0, m n=1$ and $m$.

(2) Check the stopping criteria.

(3) Let $x_{k+1}=x_{k}+\Delta_{k} C_{k}^{i}$ and compute $f\left(x_{k+1}\right)$. If $f\left(x_{k+1}\right)<f\left(x_{k}\right)$ then go to step (4), else go to step (5).

(4) If $m n \leq m$, then $x_{k+1}^{\prime}=x_{k+1}+x_{k+1}-x_{k}$ and compute $f\left(x_{k+1}^{\prime}\right)$. If $f\left(x_{k+1}^{\prime}\right)<f\left(x_{k}\right)$, then $x_{k}=x_{k+1}, \quad x_{k+1}=x_{k+1}^{\prime}, m n=m n+1$ go to step (4); else $\Delta_{k+1}^{k+1}=\Delta_{k}, \quad x_{k} \stackrel{k+1}{=} x_{k+1}, k=k+1$, go to step (5).

(5) If $i<n+1$, then $i=i+1$, go to step (3); else set

$$
x_{k+1}=x_{k}, \Delta_{k+1}=\frac{1}{2} \Delta_{k}, k=k+1, i=1 \text {, go to step }
$$

(2).

In fact, from the above algorithms, we can see that if we think any successful step as an iteration, then $B$ in our algorithm should be $I$ (identity matrix) or $P_{n}(i, j)$ (A matrix which exchanges the $i-t h$ row (or column) and the $j-t h$ one of the identity matrix). Whenever a step is found failure, then $B$ is set to be $I$ again. It is easy to know that our choices and settings satisfied the conditions in $[5,6]$. Then, we would like to state the convergence theorem which is also the same as in $[5,6]$.

Theorem 4 Assume that $L\left(x_{0}\right)$ is compact and that $f$ is continuously differentiable on a neighborhood of $L\left(x_{0}\right)$. Then for the sequence of iterates $x_{k}$ generated by algorithm 3 , we have

$\liminf _{k \rightarrow \infty}\left\|\nabla \mathrm{f}\left(\mathrm{x}_{\mathrm{k}}\right)\right\|=0$.

Proof: The reader is referred to $[5,6]$.

Remark: $L\left(x_{0}\right)$ is Level set defined as follows:

$$
L\left(x_{0}\right)=\left\{x \mid f(x) \leq f\left(x_{0}\right), x \in R^{n}\right\} \text {. }
$$

We tested our algorithm on the 18 examples given by Moré, Garbow and Hillstrom [9]. The 19-th is our testing problem at the beginning which we used for testing the effectiveness of the new algorithm. Its definition is: $f(x)=x_{1}^{2}+x_{2}^{2}$. We select $m$ to equal $0,1,2,3,5$ and 10 respectively. It is easy to know that when $m=0$, it is just the traditional pattern search method with positive basis.

The column "P" denotes the number of the problems, and " $\mathrm{N}$ " the number of variables. The numerical results are given by "F" which denotes the number of function evaluations. And "f" denotes the final function value we got when $m=2$. Additionally, the symbols " $\times$ " means that the algorithm terminates because the number of function evaluation exceeds 500,000. And for the easy comparing among the results we rearranged the order of the number of problems. The stopping condition we select is

$$
\left|\Delta_{k}\right| \leq 10^{-6} \text {, }
$$

which is different with other relative documents.

We select Equation (1) as the stopping criteria just be- cause it is simple and easy for understanding. It is thought that if very small step can not lead to decrease in function value, then the current iteration point maybe located in a neighborhood of a local minimum. The algorithm is also terminated if the number of function evaluations exceeds 500,000. And we test it from three kinds of initial points, say, $x_{0}, 10 x_{0}$ and $100 x_{0}$. The values of them are suggested in [10]. We will see that our algorithm is robust and performs the best when $m=2$. The results are represented in the following tables in the Appendix.

\section{Acknowledgements}

We are very grateful to the referees for their valuable comments and suggestions.

\section{REFERENCES}

[1] A. Ismael, F. Vaz and L. N. Vicente, "A Particle Swarm Pattern Search Method for Bound Constrained Global Optimization,” J. Glob. Optim., Vol. 39, No. 2, 2007, pp. 197-219. http://dx.doi.org/10.1007/s10898-007-9133-5

[2] S. Sankaran, C. Audet and A. L. Marsden, “A Method for Stochastic Constrained Optimization Using Derivative-Free Surrogate Pattern Search and Collocation,” J. Comput. Phys., Vol. 229, No. 12, 2010, pp. 4664-4682. http://dx.doi.org/10.1016/j.jcp.2010.03.005

[3] J. Yuan, Z. Liu and Y. Wu, "Discriminative Video Pattern Search for Efficient Action Detection,” IEEE Trans. on PAMI., Vol. 33, No. 9, 2011, pp. 1728-1743. http://dx.doi.org/10.1109/TPAMI.2011.38

[4] R. M. Lewis, V. Torczon and M. W. Trosset, "Direct Search Methods: Then and Now,” J. Comp. Appl. Math., Vol. 124, No. 1-2, 2000, pp. 191-207. http://dx.doi.org/10.1016/S0377-0427(00)00423-4

[5] V. Torczon, "On the Convergence of Pattern Search Algorithms,” SIAM J. Optim, Vol. 7, 1997, pp. 1-25. http://dx.doi.org/10.1137/S1052623493250780

[6] R. M. Lewis and V. Torczon, "Rank Ordering and Positive Bases in Pattern Search Algorithms,” Technical report TR96-71, ICASE, 2000.

[7] R. Hooke and T. A. Jeeves, "'Direct Search' Solution of Numerical and Statistical Problems,” J. ACM., Vol. 8, No. 2, 1961, pp. 212-229. http://dx.doi.org/10.1145/321062.321069

[8] C. Davis, "Theory of Positive Linear Dependence," Amer. J. Math., Vol. 76, 1954, pp. 733-746. http://dx.doi.org/10.2307/2372648

[9] V. Torczon, "Multi-Directional Search: A Direct Search Algorithm for Parallel Machines,” PH.D Thesis, Department of Mathematical sciences, Rice University, Houston, TX, 1989.

[10] J. J. Mor, B. S. Garbow and K. E. Hillstrom, "Testing Unconstrained Optimization Software,” ACM Transactions on Mathematical Software, Vol. 7, 1981, pp. 17-41. http://dx.doi.org/10.1145/355934.355936 


\section{Appendix}

Table 1. The results for initial point $x_{0}$.

\begin{tabular}{ccccccccc}
\hline $\mathrm{P}$ & $\mathrm{N}$ & $\mathrm{m}=0, \mathrm{~F}$ & $\mathrm{~m}=1, \mathrm{~F}$ & $\mathrm{~m}=2, \mathrm{~F}$ & $\mathrm{~m}=3, \mathrm{~F}$ & $\mathrm{~m}=5, \mathrm{~F}$ & $\mathrm{~m}=10, \mathrm{~F}$ & $\mathrm{~m}=2, \mathrm{f}$ \\
\hline 2 & 6 & $\times$ & $\times$ & $\times$ & $\times$ & $\times$ & $\times$ & - \\
4 & 2 & $\times$ & $\times$ & $\times$ & $\times$ & $\times$ & $\times$ & - \\
10 & 2 & $\times$ & $\times$ & $\times$ & $\times$ & $\times$ & $\times .52906 \mathrm{D}-2$ \\
12 & 3 & $\times$ & 130,552 & 127,201 & 105,761 & 119,244 & $\times$ & $4.34729 \mathrm{D}-8$ \\
1 & 3 & 5590 & 70,774 & 7741 & 7750 & 7750 & 7750 & $1.16348 \mathrm{D}-8$ \\
3 & 3 & 7182 & 53,656 & 7412 & 7413 & 7413 & 7413 & 534.653 \\
5 & 3 & 2825 & 839 & 485 & 2441 & 2419 & 2398 & $1.20174 \mathrm{D}-9$ \\
6 & 3 & 2651 & 121 & 2607 & 2639 & 2607 & 2607 & 0.47141 \\
7 & 3 & 1812 & 5140 & 895 & 1644 & 1644 & 1644 & $1.68911 \mathrm{D}-5$ \\
8 & 3 & 176,941 & 109 & 110 & 110 & 110 & 110 & $4.75148 \mathrm{D}-2$ \\
9 & 3 & 1326 & 105 & 161 & 1284 & 1284 & 1284 & 85822.2 \\
11 & 4 & 14,559 & 92,655 & 13,991 & 14011 & 14,010 & 14,010 & $2.57368 \mathrm{D}-3$ \\
13 & 3 & 709 & 651 & 665 & 670 & 670 & 670 & 4.32517 \\
14 & 3 & 13,858 & 27,322 & 20,993 & 28,958 & 95,780 & 18,656 & 2.39103 \\
15 & 3 & 8310 & 11,383 & 8403 & 8403 & 8403 & 8403 & $8.91800 \mathrm{D}-4$ \\
16 & 2 & 9658 & 20,884 & 4011 & 5058 & 4511 & 4511 & $5.087505 \mathrm{D}-12$ \\
17 & 4 & 58,246 & $\times$ & 50,618 & 50,841 & 50,876 & 50,876 & $4.36513 \mathrm{D}-12$ \\
18 & 4 & 374 & 861 & 423 & 425 & 425 & 425 & $3.63798 \mathrm{D}-12$ \\
19 & 2 & 156 & 157 & 157 & 157 & 157 & 157 & \\
\end{tabular}

Table 2. The results for initial point $10 x_{0}$.

\begin{tabular}{|c|c|c|c|c|c|c|c|c|}
\hline $\mathrm{P}$ & $\mathrm{N}$ & $\mathrm{m}=0, \mathrm{~F}$ & $\mathrm{~m}=1, \mathrm{~F}$ & $\mathrm{~m}=2, \mathrm{~F}$ & $\mathrm{~m}=3, \mathrm{~F}$ & $\mathrm{~m}=5, \mathrm{~F}$ & $\mathrm{~m}=10, \mathrm{~F}$ & $\mathrm{~m}=2, \mathrm{f}$ \\
\hline 2 & 6 & $\times$ & $\times$ & $\times$ & $\times$ & $\times$ & $\times$ & - \\
\hline 4 & 2 & $x$ & $\times$ & $\times$ & $\times$ & $x$ & $\times$ & - \\
\hline 10 & 2 & $\times$ & $\times$ & $\times$ & $\times$ & $\times$ & $\times$ & - \\
\hline 14 & 3 & $x$ & 395,663 & 405,203 & $x$ & $x$ & $\times$ & 11.85160 \\
\hline 1 & 3 & 5599 & 1494 & 7750 & 7759 & 7759 & 5194 & 4.34729D-8 \\
\hline 3 & 3 & 13,989 & $\times$ & 962 & 27,048 & 10,557 & 9172 & 0.28108 \\
\hline 5 & 3 & 87,860 & 410 & 376 & 362 & 343 & 329 & 84.988 \\
\hline 6 & 3 & 2676 & 86 & 86 & 86 & 86 & 86 & 32.4938 \\
\hline 7 & 3 & 1812 & 5165 & 918 & 1664 & 1661 & 1658 & 0.47140 \\
\hline 8 & 3 & 177,085 & 255 & 10301 & 1711 & 8963 & 240,840 & $1.65986 \mathrm{D}-5$ \\
\hline 9 & 3 & 1453 & 679 & 1605 & 1622 & 1569 & 1522 & 3.3929D-6 \\
\hline 11 & 4 & 18,145 & 112,591 & 16,484 & 788 & 16337 & 16276 & 85822.2 \\
\hline 12 & 3 & 19,716 & 5833 & 14,437 & 32,520 & 32520 & 32,520 & $8.7120 \mathrm{D}-4$ \\
\hline 13 & 3 & 717 & 708 & 762 & 767 & 767 & 767 & $2.5736 \mathrm{D}-3$ \\
\hline 15 & 3 & 8571 & 11,765 & 8571 & 8637 & 8570 & 8539 & 2.39102 \\
\hline 16 & 2 & 9685 & 20,904 & 4026 & 5074 & 4525 & 4523 & $8.91800 \mathrm{D}-4$ \\
\hline 17 & 4 & 98,178 & 78,527 & 85,858 & 86,334 & 86,388 & 86,383 & $5.087505 \mathrm{D}-7$ \\
\hline 18 & 4 & 473 & 507 & 494 & 494 & 495 & 495 & 6.13756D-11 \\
\hline 19 & 2 & 201 & 190 & 188 & 189 & 190 & 190 & 3.63798D-12 \\
\hline
\end{tabular}


Table 3. The results for initial point $100 x_{0}$.

\begin{tabular}{|c|c|c|c|c|c|c|c|c|}
\hline $\mathrm{P}$ & $\mathrm{N}$ & $\mathrm{m}=0, \mathrm{~F}$ & $\mathrm{~m}=1, \mathrm{~F}$ & $\mathrm{~m}=2, \mathrm{~F}$ & $\mathrm{~m}=3, \mathrm{~F}$ & $\mathrm{~m}=5, \mathrm{~F}$ & $\mathrm{~m}=10, \mathrm{~F}$ & $\mathrm{~m}=2, \mathrm{f}$ \\
\hline 5 & 3 & $\times$ & 101 & 98 & 95 & 95 & 92 & 10222 \\
\hline 10 & 2 & $\times$ & $x$ & $\times$ & $\times$ & $x$ & $x$ & - \\
\hline 14 & 3 & $\times$ & $\times$ & $\times$ & $\times$ & $x$ & $x$ & - \\
\hline 1 & 3 & 5689 & 1584 & 7840 & 7849 & 7849 & 7849 & 403472D-8 \\
\hline 2 & 6 & 142,287 & 66,879 & 59,418 & 58,982 & 51,958 & 51,426 & 8.70496 \\
\hline 3 & 3 & 856 & 1167 & 393 & 352 & 463 & 430 & 0.41085 \\
\hline 4 & 2 & 134 & 152 & 152 & 152 & 152 & 152 & 1.000 \\
\hline 6 & 3 & 4375 & 35,350 & 1405 & 9375 & 3863 & 5833 & 91.756 \\
\hline 7 & 3 & 2212 & 5390 & 1098 & 1819 & 1796 & 1775 & 0.47140 \\
\hline 8 & 3 & 178,525 & 1245 & 11,067 & 2470 & 9622 & 241,459 & $1.6598 \mathrm{D}-5$ \\
\hline 9 & 3 & 1633 & 789 & 1695 & 1700 & 1638 & 1579 & 3.392D-6 \\
\hline 11 & 4 & 50,455 & 162,471 & 61,685 & 32,205 & 29,500 & 27,933 & 85822.2 \\
\hline 12 & 3 & 81 & 81 & 81 & 81 & 81 & 81 & 32.835 \\
\hline 13 & 3 & 608 & 1042 & 1091 & 1095 & 1095 & 1095 & $2.5736 \mathrm{D}-3$ \\
\hline 15 & 3 & 11181 & 29,597 & 9944 & 10,002 & 9706 & 10,387 & 2.391 \\
\hline 16 & 2 & 9955 & 21,084 & 4176 & 5208 & 4645 & 4628 & 8.91800D-4 \\
\hline 17 & 4 & 133,549 & 88,233 & 115,142 & 108,730 & 115,674 & 115,623 & $5.087505 \mathrm{D}-7$ \\
\hline 18 & 4 & 1013 & 869 & 977 & 768 & 868 & 724 & 6.13756D-11 \\
\hline 19 & 2 & 651 & 505 & 458 & 434 & 415 & 394 & 3.63798D-12 \\
\hline
\end{tabular}

